\title{
Left Oophorectomy
}

National Cancer Institute

\section{Source}

National Cancer Institute. Left Oophorectomy. NCI Thesaurus. Code C51601.

Surgery to remove the left ovary. 